\title{
A Robust Algorithm for Extracting Signals with Temporal Structure
}

\author{
Yibing $\mathrm{LI}^{\dagger \mathrm{a})}$, Nonmember, Wei $\mathrm{NIE}^{\dagger \mathrm{b})}$, Student Member, and Fang $\mathrm{YE}^{\dagger \mathrm{c})}$, Nonmember
}

\begin{abstract}
SUMMARY The separation of signals with temporal structure from mixed sources is a challenging problem in signal processing. For this problem, blind source extraction (BSE) is more suitable than blind source separation (BSS) because it has lower computation cost. Nowadays many BSE algorithms can be used to extract signals with temporal structure. However, some of them are not robust because they are too dependent on the estimation precision of time delay; some others need to choose parameters before extracting, which means that arbitrariness can't be avoided. In order to solve the above problems, we propose a robust source extraction algorithm whose performance doesn't rely on the choice of parameters. The algorithm is realized by maximizing the objective function that we develop based on the non-Gaussianity and the temporal structure of source signals. Furthermore, we analyze the stability of the algorithm. Simulation results show that the algorithm can extract the desired signal from large numbers of observed sensor signals and is very robust to error in the estimation of time delay.

key words: blind source extraction, non-Gaussianity, temporal structure, time delay
\end{abstract}

\section{Introduction}

In recent years, BSS has been attracting more attention because its processing performance is satisfactory. It has been successfully applied in digital image processing [1], remote sensing image processing [2], machinery fault diagnostic [3], speech signal processing [4], biomedical signal processing [5] and so on. Blind source separation techniques can separate all mixed signals into independent source signals. However, sometimes we just want to focus on one or a few signals which are of interest. What's more, such signals are usually featured with some special properties that can be utilized as prior information. Therefore, BSE technique has been developed to reduce the time taken to separate all source signals from a large number of observed sensor signals. In practice, many kinds of signals have special temporal structure which is utilized by blind source extraction technique to extract the desired source signals.

Large numbers of BSE algorithms based on temporal structure have been proposed. Barros and Cichocki [6] developed an efficient algorithm that is based on the autocorrelation property of the desired source signals. This algorithm needs the proper selection of the time delay. In other

Manuscript received October 8, 2015.

Manuscript revised January 12, 2016.

Manuscript publicized March 15, 2016.

${ }^{\dagger}$ The authors are with School of Information and Communication Engineering, Harbin Engineering University, Harbin, China.

a) E-mail: liyibing0920@ sina.cn

b) E-mail: niewei0207@ sina.cn

c) E-mail: yefang0815@sina.cn (Corresponding author)

DOI: $10.1587 /$ transinf.2015EDP7420 words, the performance of the algorithm depends on the estimation precision of the time delay, a deficiency that limits its practical application. Zhang et al. [7] extended Barros's algorithm and proposed another efficient algorithm based on eigenvalue decomposition. The algorithm works out the satisfactory performance when the estimation error of the time delay is acceptable. Shi et al. [8] proposed a quite robust algorithm, but the performance of the algorithm is affected by the selection of parameters. Li et al. [9] considered the effect of noise on the basis of Zhang's algorithm. However, the estimation accuracy needs to be guaranteed like Zhang's algorithm.

In this paper, we propose a robust algorithm based on the non-Gaussianity and the temporal structure of source signals. The algorithm offers improved robustness to the time delay and, unlike some other algorithms, avoids any dependency on parameter setting. Theoretical analysis and simulation results confirm the algorithm's robustness and practicability.

\section{The Signal Model}

Let the observed signals $\mathbf{x}(t)=\left[x_{1}(t), x_{2}(t), \ldots, x_{N}(t)\right]^{T}$ be linear combinations of the source signals $\mathbf{s}(t)=$ $\left[s_{1}(t), s_{2}(t), \ldots, s_{N}(t)\right]^{T}$ as:

$$
\mathbf{x}(t)=\mathbf{A s}(t)
$$

where $\mathbf{A}$ is an $N \times N$ unknown non-singular matrix and source signals own zero-mean and unit-variance. A prewhitening method is usually adopted to transform the observed signals $\mathbf{x}$ into the whitened signals $\tilde{\mathbf{x}}=\mathbf{V x}, E\left\{\tilde{\mathbf{x}} \tilde{\mathbf{x}}^{T}\right\}=$ $\mathbf{I}$, where $\mathbf{V}$ is the corresponding pre-whitening matrix. Assume the estimated source signals can be represented as a matrix $\mathbf{y}(t)=\left[y_{1}(t), y_{2}(t), \ldots, y_{N}(t)\right]^{T}$, the target of BSS is to define $\mathbf{y}(t)$ by finding an $N \times N$ unmixing matrix $\mathbf{W}=$ $\left(\mathbf{w}_{1}, \mathbf{w}_{2}, \ldots, \mathbf{w}_{N}\right)$, that is

$$
\mathbf{y}(t)=\mathbf{W} \tilde{\mathbf{x}}(t)
$$

Similarly, the target of BSE is to find an $1 \times N$ unmixing vector $\mathbf{w}_{i}^{T}$ which satisfies $y_{i}(t)=\mathbf{w}_{i}{ }^{T} \tilde{\mathbf{x}}(t)$. In Sect. 3, 1-D signal $y(t)$ is assumed.

We assume that the source signals have linear autocorrelation and satisfy the following conditions for a positive integer time delay $\tau$ :

$$
\left\{\begin{aligned}
E\left\{s_{i} s_{i, \tau}\right\} & =E\left\{s_{i}(t) s_{i}(t-\tau)\right\} \neq 0 \\
E\left\{s_{i} s_{j, \tau}\right\} & =E\left\{s_{i}(t) s_{j}(t-\tau)\right\}=0
\end{aligned}\right.
$$

where $j \neq i$. 


\section{Algorithms Description}

\subsection{Barros's Algorithm}

Barros's algorithm defined the following error function

$$
\varepsilon(t)=y(t)-b y(t-\tau)
$$

where $b$ is a parameter. Then, the mean squared error $\xi(\mathbf{w}, b)=E\left[\varepsilon(t)^{2}\right]$ was designed as the objective function. Through mathematical derivation, $\xi(\mathbf{w}, b)$ can be denoted as

$$
\begin{aligned}
& \xi(\mathbf{w}, b)=\mathbf{w}^{T} E\left[\tilde{\mathbf{x}}(t) \tilde{\mathbf{x}}(t)^{T}\right] \mathbf{w}-2 b \mathbf{w}^{T} E[\tilde{\mathbf{x}}(t) \\
& \left.\tilde{\mathbf{x}}(t-\tau)^{T}\right] \mathbf{w}+b^{2} \mathbf{w}^{T} E\left[\tilde{\mathbf{x}}(t-\tau) \tilde{\mathbf{x}}(t-\tau)^{T}\right] \mathbf{w}=0
\end{aligned}
$$

The above function reaches minimum when its gradient reaches zero in relation to $\mathbf{w}$ and $b$. Set the following two equations

$$
\begin{aligned}
& \frac{\partial \xi(\mathbf{w}, b)}{\partial \mathbf{w}}=2 E\left[\tilde{\mathbf{x}}(t) \tilde{\mathbf{x}}(t)^{T}\right] \mathbf{w}-4 b E\left[\tilde{\mathbf{x}}(t) \tilde{\mathbf{x}}(t-\tau)^{T}\right] \mathbf{w} \\
& +2 b^{2} E\left[\tilde{\mathbf{x}}(t-\tau) \tilde{\mathbf{x}}(t-\tau)^{T}\right] \mathbf{w}=0 \\
& \frac{\partial \xi(\mathbf{w}, b)}{\partial b}=2 b \mathbf{w}^{T} E\left[\tilde{\mathbf{x}}(t-\tau) \tilde{\mathbf{x}}(t-\tau)^{T}\right] \mathbf{w} \\
& -2 \mathbf{w}^{T} E\left[\tilde{\mathbf{x}}(t) \tilde{\mathbf{x}}(t-\tau)^{T}\right] \mathbf{w}=0
\end{aligned}
$$

We know the equation $E\left[\tilde{\mathbf{x}}(t) \tilde{\mathbf{x}}(t)^{T}\right]=\mathbf{I}$, so the following learning rule can be simplified as

$$
\mathbf{w}=\frac{2 b}{1+b^{2}} E\left[\tilde{\mathbf{x}}(t) \tilde{\mathbf{x}}(t-\tau)^{T}\right] \mathbf{w}
$$

To avoid the situation $\mathbf{w}=\mathbf{0}, \mathbf{w}=\mathbf{w} /\|\mathbf{w}\|$ is used at each iteration. Because of the normalization, the term $2 b /\left(1+b^{2}\right)$ can be ignored. Finally, Barros's algorithm can be concluded as

$$
\left\{\begin{array}{c}
\mathbf{w}=E\left[\tilde{\mathbf{x}}(t) \tilde{\mathbf{x}}(t-\tau)^{T}\right] \mathbf{w} \\
\mathbf{w}=\mathbf{w} /\|\mathbf{w}\|
\end{array}\right.
$$

\subsection{Zhang's Algorithm}

Zhang first proposed an objective function as follows

$$
J(\mathbf{w})=E[y(t) y(t-\tau)]=\mathbf{w}^{T} E\left[\tilde{\mathbf{x}}(t) \tilde{\mathbf{x}}(t-\tau)^{T}\right] \mathbf{w}
$$

The algorithm got the desired signal by maximizing $J(\mathbf{w})$ and described the reason in [7]. We can sum up the reason in [7] as follows. The paper researched the signal with temporal structure. For the desired source signal, this autocorrelation should have a high value, while this value should be very small for other source signals.

Then, the authors designed the following objective function based on (10) in order to avoid the iterative process in Barros's algorithm.

$$
\begin{aligned}
& J_{1}(\mathbf{w})=\frac{1}{2} J(\mathbf{w})+\frac{1}{2} J(\mathbf{w})^{T}= \\
& \frac{1}{2} \mathbf{w}^{T} E\left[\tilde{\mathbf{x}}(t) \tilde{\mathbf{x}}(t-\tau)^{T}\right] \mathbf{w}+\frac{1}{2} \mathbf{w}^{T} E\left[\tilde{\mathbf{x}}(t-\tau) \tilde{\mathbf{x}}(t)^{T}\right] \mathbf{w} \\
& =\frac{1}{2} \mathbf{w}^{T}\left(\mathbf{R}_{\tilde{\mathbf{x}}}(\tau)+\mathbf{R}_{\tilde{\mathbf{x}}}(\tau)^{T}\right) \mathbf{w}
\end{aligned}
$$

$J_{1}(\mathbf{w})$ implies that the maximization of the Eq. (11) under the constraint $\|\mathbf{w}\|=1$ is equivalent to finding the eigenvector corresponding to the maximal eigenvalue of the real symmetric matrix $\mathbf{R}=\mathbf{R}_{\tilde{\mathbf{x}}}(\tau)+\mathbf{R}_{\tilde{\mathbf{x}}}(\tau)^{T}$, where $\mathbf{R}_{\tilde{\mathbf{x}}}(\tau)=$ $E\left[\mathbf{x}(t) \mathbf{x}(t-\tau)^{T}\right]$. Based on the above theory, the following algorithm is obtained

$$
\left\{\begin{array}{c}
\mathbf{R}_{\tilde{\mathbf{x}}}(\tau)=E\left[\tilde{\mathbf{x}}(t) \tilde{\mathbf{x}}(t-\tau)^{T}\right] \\
\mathbf{w}=E I G\left(\mathbf{R}_{\tilde{\mathbf{x}}}(\tau)+\mathbf{R}_{\tilde{\mathbf{x}}}(\tau)^{T}\right)
\end{array}\right.
$$

where $E I G(\cdot)$ represents the eigenvalue decomposition operator. It can get the unmixing vector for the desired source signal through the normalized eigenvector for the maximal eigenvalue. In order to improve the robustness, Zhang's algorithm is eventually written as

$$
\left\{\begin{array}{c}
\mathbf{R}_{\tilde{\mathbf{x}}}(\tau)=E\left[\tilde{\mathbf{x}}(t) \tilde{\mathbf{x}}(t-\tau)^{T}\right] \\
\mathbf{W}=E I G\left(\sum_{k=1}^{K} \mathbf{R}_{\tilde{\mathbf{x}}}(k \tau)+\mathbf{R}_{\tilde{\mathbf{x}}}(k \tau)^{T}\right)
\end{array}\right.
$$

where $K$ is a positive integer.

\subsection{Shi’s Algorithm}

In Shi's algorithm, the non-Gaussianity is utilized. NonGaussianity is opposite to Gaussianity. In ICA models, non-Gaussianity has large connection with independence. Central-limit theorem [10] is a classical theory of probability theory. The theorem tells us that the distribution of the sum of independent random variables tends to Gaussian distribution under certain conditions. We can consider that the distribution of the sum of two independent random variables is closer to the Gaussian distribution than any of two original random variables. Here, we take an example. Assuming $y=\mathbf{b}^{T} \mathbf{x}$, we observe the non-Gaussian degree of $y$ when b changes. We can find the optimum face has $2 n$ local extremum in the $n$ dimensions space that is corresponding to b. For each independent component, there are two local extremum points that are corresponding to $s_{i}$ and $-s_{i}$ respectively. The independent signal can be got by maximizing non-Gaussian. In other words, The independence can be obtained by non-Gaussian.

Utilizing the error function $\varepsilon(t)=y(t)-b y(t-\tau)$ in Barros's algorithm and combining the non-Gaussianity, Shi presented the following constrained minimization problem:

$$
\min _{\|\mathbf{w}\|=1} E[G(\varepsilon(t))]=E[G(\mathbf{w}(\tilde{\mathbf{x}}(t)-b \tilde{\mathbf{x}}(t-\tau)))]
$$

where $G$ is a differentiable function. The objective function that consists of $\mathrm{G}$ can measure the non-Gaussianity. According to the gradient of $G(\varepsilon(t))$ with respect to $\mathbf{w}$ and $b$, a learning algorithm is got as

$$
\begin{aligned}
& \mathbf{w} \leftarrow \mathbf{w}-\mu_{\mathbf{w}} \frac{\partial E[G(\varepsilon(t))]}{\partial \mathbf{w}}=\mathbf{w}-\mu_{\mathbf{w}}(E[(\tilde{x}(t) \\
& \left.\left.-b \tilde{x}(t-\tau)) g\left(\mathbf{w}^{T}(\tilde{x}(t)-b \tilde{x}(t-\tau))\right)\right]\right) \\
& b \leftarrow b-\mu_{\mathbf{w}} \frac{\partial E[G(\varepsilon(t))]}{\partial b}=b+\mu_{b}\left(E \left[\mathbf{w}^{T} \tilde{\mathbf{x}}(t-\tau)\right.\right. \\
& \left.\left.g\left(\mathbf{w}^{T}(\tilde{\mathbf{x}}(t)-b \tilde{\mathbf{x}}(t-\tau))\right)\right]\right) \\
& \mathbf{w}=\mathbf{w} /\|\mathbf{w}\|
\end{aligned}
$$


where $g$ is the derivative of $G, \mu_{w}$ and $\mu_{b}$ are learning rates.

\subsection{The Proposed Algorithm}

\subsubsection{Objective Function}

In order to ensure that all components of $\tilde{\mathbf{x}}$ are uncorrelated and featured with unit variance, we transform the observed signals $\mathbf{x}$ into $\tilde{\mathbf{x}}=\mathbf{V x}$ through the pre-whitening matrix. Such a pre-whitening strategy decreases the difficulty of the problem.

Though the objective function in Barros's algorithm includes $b$, the learning algorithm is uncorrelated with $b$. Therefore, we don't need to choose the initial value of $b$. But this objective function is not robust. Comparing with Barros's algorithm, Zhang's algorithm improves the performance but is still not enough robust. Shi's algorithm utilizes the non-Gaussianity based on Barros's algorithm. If only the non-Gaussianity is utilized, the extracted independent signal may be not the desired signal. In order to improve it, Shi combines non-Gaussianity and time-correlation to get the desired independent signal. However, the initial value of $b$ and two learning rates included in the learning algorithm of Shi should be set because of utilizing the gradient algorithm. Setting improper parameters will reduce the performance of the algorithm and make the algorithm time-consuming. In a word, the performance of the algorithm is up to the choice of parameters. But Shi's algorithm improves the robustness when the proper parameters are chosen. The objective function in [8] measures the non-Gaussianity of the error function. The simulation in [8] suggests adding $G$ to the problem can make the algorithm more robust. We analyze its theory. After adding $G$, the objective function in [8] can get the extremum even if there exists larger difference between the estimated time delay and the optimal time delay. In other words, comparing with the original objective function, the introduction of $G$ will reduce the sensitivity of the algorithm to the estimation of the optimal time delay.

Considering the similar theory, we can also introduce $G$ to get robustness like [8]. In order to avoid the blindness of setting parameters and make the algorithm more robust, we combine the non-Gaussianity in Shi's algorithm and the correlation function in Zhang's algorithm, and then design a new objective function

$$
\begin{aligned}
& E\{G[y(t)] G[y(t) y(t-\tau)]\}=E\left\{G\left[\mathbf{w}^{T} \tilde{\mathbf{x}}(t)\right]\right. \\
& \left.G\left[\mathbf{w}^{T} \tilde{\mathbf{x}}(t) \tilde{\mathbf{x}}(t-\tau)^{T} \mathbf{w}\right]\right\}
\end{aligned}
$$

This function combines the non-Gaussianity of the correlation function of the estimated signal and the delayed estimated signal with the non-Gaussianity of the estimated signal. It also utilizes non-Gaussianity and time-correlation like Shi's algorithm. When the extracted signal is the source signal, $E\{G[y(t)]\}$ can reach a large extremum. However, the extracted signal may be not the desired signal. In order to compensate the shortage, $G[y(t) y(t-\tau)]$ is introduced. When the extracted signal is the desired source signal, the objective function can reach a large extremum. But when the extracted signal is not the desired source signal, the objective function can't reach a large extremum because $G[y(t) y(t-\tau)]$ is related with temporal structure of the desired signal. Specifically two terms in the expression can't be large values simultaneously. We need to maximize the above function under the constraint $\|\mathbf{w}\|=1$ and choose a proper function $G$ by utilizing the probability distribution of the innovation [11]-[13].

\subsubsection{Learning Algorithm}

With respect to $\mathbf{w}$, the gradient of $E\{G[y(t)] G[y(t) y(t-\tau)]\}$ can be represented as:

$$
\begin{aligned}
& \frac{\partial E\{G[y(t)] G[y(t) y(t-\tau)]\}}{\partial \mathbf{w}}=E\{\tilde{\mathbf{x}}(t) g[y(t)] G[y(t) \\
& y(t-\tau)]\}+E\{\tilde{\mathbf{x}}(t) y(t-\tau) g[y(t) y(t-\tau)] \\
& G[y(t)]\}+E\{\tilde{\mathbf{x}}(t-\tau) y(t) g[y(t) y(t-\tau)] G[y(t)]\}
\end{aligned}
$$

According to the gradient ascent learning rule, we can maximize the objective function (18) and derive a gradient method based on (19).

$$
\left\{\begin{array}{c}
\mathbf{w} \leftarrow \mathbf{w}+u \frac{\partial E\{G[y(t)] G[y(t) y(t-\tau)]\}}{\partial \mathbf{w}} \\
=\mathbf{w}+u(E\{\tilde{\mathbf{x}}(t) g[y(t)] G[y(t) y(t-\tau)]\} \\
+E\{\tilde{\mathbf{x}}(t) y(t-\tau) g[y(t) y(t-\tau)] G[y(t)]\} \\
+E\{\tilde{\mathbf{x}}(t-\tau) y(t) g[y(t) y(t-\tau)] G[y(t)]\}) \\
\mathbf{w}=\mathbf{w} /\|\mathbf{w}\|
\end{array}\right.
$$

where $\mu$ is a learning rate. We note that the gradient must point in the direction of $\mathbf{w}$ at a stable point of the gradient algorithm. It means the gradient must be equal to $\mathbf{w}$ multiplied by some scalar constant. Only in such a case, adding the gradient to $\mathbf{w}$ does not change its direction and the convergence can be got. Through normalization to the unit norm, the value of $\mathbf{w}$ is not changed except perhaps by changing its sign. Therefore, we can obtain

$$
\begin{aligned}
& w \propto E\{\tilde{\mathbf{x}}(t) g[y(t)] G[y(t) y(t-\tau)]\} \\
& +E\{\tilde{\mathbf{x}}(t) y(t-\tau) g[y(t) y(t-\tau)] G[y(t)]\} \\
& +E\{\tilde{\mathbf{x}}(t-\tau) y(t) g[y(t) y(t-\tau)] G[y(t)]\}
\end{aligned}
$$

Based on the above formula, a fixed-point algorithm can be described as

$$
\left\{\begin{array}{c}
\mathbf{w} \leftarrow E\{\tilde{\mathbf{x}}(t) g[y(t)] G[y(t) y(t-\tau)]\} \\
+E\{\tilde{\mathbf{x}}(t) y(t-\tau) g[y(t) y(t-\tau)] G[y(t)]\} \\
+E\{\tilde{\mathbf{x}}(t-\tau) y(t) g[y(t) y(t-\tau)] G[y(t)]\} \\
\mathbf{w}=\mathbf{w} /\|\mathbf{w}\|
\end{array}\right.
$$

The derivative $g$ of each function $G$ is different. Different values of $g$ will lead to different results. Therefore, we need to choose a proper $g$. The theoretical analysis and the criteria for the choice of $G$ and $g$ are described in [14] and [15]. The function $g$ should be chosen according to the probability distribution of the residual instead of the actual component. If the residual is supergaussian, $g(u)=u+\tanh (u)$ in [14] or $g(u)=\tanh (a u)$ in [15] is adopted, where $a$ is a suitable constant that is greater than 1. For subgaussian residuals, $g(u)=u-\tanh (u)$ in [14] or $g(u)=u^{3}$ in [15] is chosen. The signal in this paper is supergaussian, so we 
must choose $g(u)=u+\tanh (u)$ or $g(u)=\tanh (a u)$. If we choose $g(u)=\tanh (a u), a$ must be set. Because different values of $a$ will result in different results, we choose $g(u)=u+\tanh (u)$ in the following simulation to avoid the effect of choosing the value of $a$.

\subsubsection{Stability and Efficiency Analysis}

We give the above learning algorithm, but some people may doubt whether the desired independent signal will be got stably by our algorithm. Actually, we can prove that we can get the desired signal stably if some weaker assumptions are satisfied. Here, we analyze the stability conditions of our algorithm and give the following theorem.

Theorem: Assume that the input data meet the ICA model as (1). The data are pre-whitened through the equation $\tilde{\mathbf{x}}=$ VAs and $G$ is a quite smooth even function. Furthermore, we assume that $\left\{s_{i}, s_{i \tau}\right\}$ and $\left\{s_{j}, s_{j \tau}\right\}$ are mutually independent. The assumption means that either of $\left\{s_{i}, s_{i \tau}\right\}$ and either of $\left\{s_{j}, s_{j \tau}\right\}$ are mutually independent. If the corresponding desired source signal $s_{i}$ satisfies:

$$
\begin{aligned}
& E\left[g^{\prime}\left(s_{i}\right) G\left(s_{i} s_{i \tau}\right)+s_{i \tau} g\left(s_{i}\right) g\left(s_{i} s_{i \tau}\right)+\right. \\
& 2 s_{i}^{2} g^{\prime}\left(s_{i} s_{i \tau}\right) G\left(s_{i}\right)+2 s_{i} s_{i \tau} s_{j} s_{j \tau} g^{\prime}\left(s_{i} s_{i \tau}\right) \\
& G\left(s_{i}\right)+3 s_{i} s_{j} s_{j \tau} g\left(s_{i}\right) g\left(s_{i} s_{i \tau}\right)-s_{i} g\left(s_{i}\right) \\
& \left.G\left(s_{i} s_{i \tau}\right)-2 s_{i} s_{i \tau} g\left(s_{i} s_{i \tau}\right) G\left(s_{i}\right)\right]<0(\forall i \neq j)
\end{aligned}
$$

the objective function $E\{G[y(t)] G[y(t) y(t-\tau)]\}$ can reach a local maximum value when extracting a source signal.

Through maximizing the objective function, we can get the desired signal. The condition ensures that the algorithm can extract the desired signal. If the condition can't be satisfied, the desired signal may not be got. It means the algorithm is not stable. However, we hope to get the desired signal stably, so the stability condition is important. The following content is the proof for the theorem.

Proof: $\left\{s_{i}, s_{i \tau}\right\}$ correspond to a source signal. $\left\{s_{j}, s_{j \tau}\right\}$ correspond to another source signal. The assumption that different source signals are mutually independent is a basic assumption in ICA and most blind source separation algorithms. Therefore, the assumption that $\left\{s_{i}, s_{i \tau}\right\}$ and $\left\{s_{j}, s_{j \tau}\right\}$ are mutually independent is mild. According to the above conditions, make the orthogonal transform of coordinates $\mathbf{q}=\mathbf{A}^{T} \mathbf{V}^{T} \mathbf{w}$, then we obtain

$$
H(\mathbf{q})=E\left\{G\left(\mathbf{q}^{T} \mathbf{s}\right) G\left(\mathbf{q}^{T} \mathbf{s} \mathbf{s}_{\tau}^{T} \mathbf{q}\right)\right\}
$$

We analyze the stability of the point $\mathbf{q}=\mathbf{e}_{1}$, where $\mathbf{e}_{1}=(1,0,0,0, \ldots)^{T}$. The independency assumptions are utilized to evaluate the gradient and the Hessian matrix of $H(\mathbf{q})$ at the point $\mathbf{q}=\mathbf{e}_{1}$. Then, through making a small perturbation $\varepsilon=\left(\varepsilon_{1}, \varepsilon_{2}, \varepsilon_{3}, \ldots\right)$ where $\varepsilon_{1}, \varepsilon_{2}$ and $\varepsilon_{3}$ are the elements of $\varepsilon$, we get

$$
\begin{aligned}
& H\left(\mathbf{e}_{1}+\varepsilon\right)=H\left(\mathbf{e}_{1}\right)+\varepsilon^{T} \frac{\partial H\left(\mathbf{e}_{1}\right)}{\partial \mathbf{q}}+\frac{1}{2} \varepsilon^{T} \frac{\partial^{2} H\left(\mathbf{e}_{1}\right)}{\partial \mathbf{q}^{2}} \varepsilon \\
& +\mathrm{O}\left(\|\varepsilon\|^{2}\right)
\end{aligned}
$$

where $\frac{\partial H\left(\mathbf{e}_{1}\right)}{\partial \mathbf{q}}$ and $\frac{\partial^{2} H\left(\mathbf{e}_{1}\right)}{\partial \mathbf{q}^{2}}$ are represented as follows:

$$
\begin{aligned}
& \frac{\partial H\left(\mathbf{e}_{1}\right)}{\partial \mathbf{q}}=\mathbf{e}_{1} E\left\{s_{1} g\left(s_{1}\right) G\left(s_{1} s_{1 \tau}\right)\right. \\
& \left.+2 s_{1} s_{1 \tau} g\left(s_{1} s_{1 \tau}\right) G\left(s_{1}\right)\right\} \\
& \frac{\partial H^{2}\left(\mathbf{e}_{1}\right)}{\partial \mathbf{q}^{2}}=\operatorname{diag}\left(E \left\{s_{1}^{2} g^{\prime}\left(s_{1}\right) G\left(s_{1} s_{1 \tau}\right)+\right.\right. \\
& \left.4 s_{1}^{2} s_{1 \tau} g\left(s_{1}\right) g\left(s_{1} s_{1 \tau}\right)+4 s_{1}{ }^{2} s_{1 \tau}{ }^{2} g^{\prime}\left(s_{1} s_{1 \tau}\right) G\left(s_{1}\right)\right\}, \\
& E\left\{g^{\prime}\left(s_{1}\right) G\left(s_{1} s_{1 \tau}\right)+s_{1 \tau} g\left(s_{1}\right) g\left(s_{1} s_{1 \tau}\right)+2 s_{1}^{2}\right. \\
& g^{\prime}\left(s_{1} s_{1 \tau}\right) G\left(s_{1}\right)+2 s_{1} s_{1 \tau} s_{2} s_{2 \tau} g^{\prime}\left(s_{1} s_{1 \tau}\right) G\left(s_{1}\right)+ \\
& \left.3 s_{1} s_{2} s_{2 \tau} g\left(s_{1}\right) g\left(s_{1} s_{1 \tau}\right)\right\}, \ldots, E\left\{g^{\prime}\left(s_{1}\right) G\left(s_{1} s_{1 \tau}\right)+\right. \\
& s_{1 \tau} g\left(s_{1}\right) g\left(s_{1} s_{1 \tau}\right)+2 s_{1}{ }^{2} g^{\prime}\left(s_{1} s_{1 \tau}\right) G\left(s_{1}\right)+2 s_{1} s_{1 \tau} \\
& \left.\left.s_{j} s_{j \tau} g^{\prime}\left(s_{1} s_{1 \tau}\right) G\left(s_{1}\right)+3 s_{1} s_{j} s_{j \tau} g\left(s_{1}\right) g\left(s_{1} s_{1 \tau}\right)\right\}\right)
\end{aligned}
$$

We can obtain $\varepsilon_{1}=\sqrt{1-\varepsilon_{2}^{2}-\varepsilon_{3}^{2}-\cdots}-1$ under the constraint $\|\mathbf{w}\|=1$. Due to $\sqrt{1-\gamma}=1-\gamma / 2+o(\gamma)$, the term of order $\varepsilon_{1}^{2}$ is $o\left(\|\varepsilon\|^{2}\right)$, namely we can neglect the higher order terms. Through the first-order approximation of $\varepsilon_{1}$, we have $\varepsilon_{1}=-\sum_{i>1} \varepsilon_{i}{ }^{2} / 2+o\left(\|\varepsilon\|^{2}\right)$, then we obtain

$$
\begin{aligned}
& H\left(\mathbf{e}_{1}+\varepsilon\right)=H\left(\mathbf{e}_{1}\right)+\frac{1}{2} E\left\{g^{\prime}\left(s_{1}\right) G\left(s_{1} s_{1 \tau}\right)+s_{1 \tau}\right. \\
& g\left(s_{1}\right) g\left(s_{1} s_{1 \tau}\right)+2 s_{1}^{2} g^{\prime}\left(s_{1} s_{1 \tau}\right) G\left(s_{1}\right)+2 s_{1} s_{1 \tau} s_{j} s_{j \tau} \\
& g^{\prime}\left(s_{1} s_{1 \tau}\right) G\left(s_{1}\right)+3 s_{1} s_{j} s_{j \tau} g\left(s_{1}\right) g\left(s_{1} s_{1 \tau}\right)-s_{1} g\left(s_{1}\right) \\
& \left.G\left(s_{1} s_{1 \tau}\right)-2 s_{1} s_{1 \tau} g\left(s_{1} s_{1 \tau}\right) G\left(s_{1}\right)\right\} \sum_{j>1} \varepsilon_{j}^{2}+o\left(\|\varepsilon\|^{2}\right)
\end{aligned}
$$

When the source signal meets the following condition

$$
\begin{aligned}
& E\left[g^{\prime}\left(s_{i}\right) G\left(s_{i} s_{i \tau}\right)+s_{i \tau} g\left(s_{i}\right) g\left(s_{i} s_{i \tau}\right)+\right. \\
& 2 s_{i}{ }^{2} g^{\prime}\left(s_{i} s_{i \tau}\right) G\left(s_{i}\right)+2 s_{i} s_{i \tau} s_{j} s_{j \tau} g^{\prime}\left(s_{i} s_{i \tau}\right) \\
& G\left(s_{i}\right)+3 s_{i} s_{j} s_{j \tau} g\left(s_{i}\right) g\left(s_{i} s_{i \tau}\right)-s_{i} g\left(s_{i}\right) \\
& \left.G\left(s_{i} s_{i \tau}\right)-2 s_{i} s_{i \tau} g\left(s_{i} s_{i \tau}\right) G\left(s_{i}\right)\right]<0(\forall i \neq j)
\end{aligned}
$$

we can know that $H\left(\mathbf{e}_{1}\right)$ is greater than $H\left(\mathbf{e}_{1}+\varepsilon\right)$ when $i$ is equal to 1 . In other words, the objective function gets a local maximum value and the value is $H\left(\mathbf{e}_{1}\right)$.

Through the above proof, we can clearly know that $\mathbf{q}=$ $\mathbf{e}_{1}$ is an extremum, and the type of the extremum is the one we need according to the condition of the theorem. We reach the conclusion that the local maxima of $E\{G[y(t)] G[y(t) y(t-$ $\tau)$ ] includes one row of the inverse of the matrix VA under the constraint $\|\mathbf{w}\|=1$. In a word, the objective function can reach a local maximum value and help us get the desired signal if the source signal meets certain conditions.

\section{Simulation Results}

We choose the data in the ABio.mat file [16] as the simulation data. The signals in the second, the fourth and the seventh channel of the data are taken as three source signals. Figure 1 shows the source signals. The sources are mixed with a $3 \times 3$ mixing matrix. In the simulation part, we set the mixing matrix as

$$
\mathbf{A}=\left[\begin{array}{lll}
0.6697 & 0.8216 & 0.8666 \\
0.2649 & 0.6600 & 0.7301 \\
0.9413 & 0.2529 & 0.8006
\end{array}\right]
$$

Through mixing, the mixed signals are shown in Fig. 2 .

The proper time delay should be got before beginning the algorithm. As described in [6], a simple solution is to 

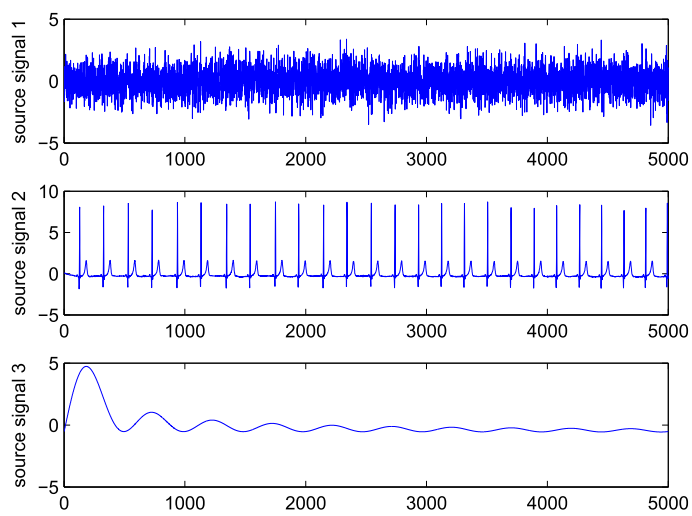

Fig. 1 Three source signals in the ABio.mat file
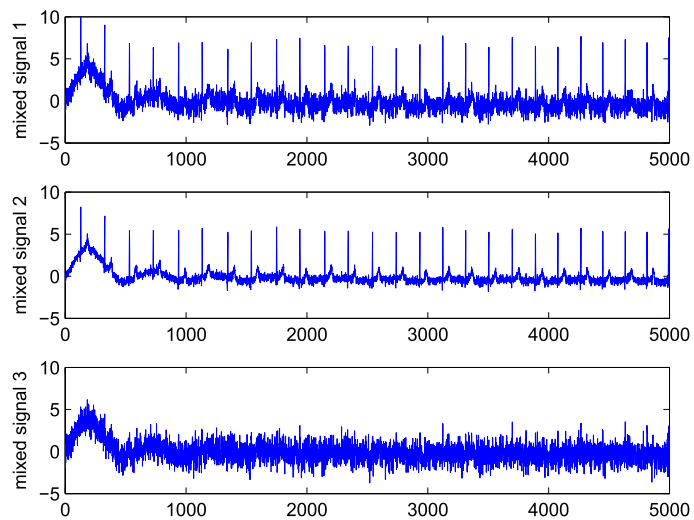

Fig. 2 The mixed signals that are mixed by three source signals

calculate the autocorrelation $E\left\{x_{i}(t) x_{i}(t-\tau)\right\}$ of the sensor signal as a function of the time delay $\tau$ and find the one corresponding to the peak within an specified interval of interest. The method regards $\tau$ as the optimal time delay when the peak appears. However, the estimation error can not be avoided in practical condition. Therefore, the correlation coefficient is proposed to compare different algorithms. The computational formula of correlation coefficient is represented as:

$$
\rho=\frac{E\left\{s_{i}(t) \hat{s}_{i}(t)\right\}}{\sqrt{E\left\{s_{i}^{2}(t)\right\}} \sqrt{E\left\{\hat{s}_{i}^{2}(t)\right\}}}
$$

where $\hat{s}_{i}(t)$ is the estimated signal.

In order to verify that our algorithm owns better robustness and extraction accuracy, we choose the second of three source signals as the desired signal and compare the correlation coefficients of different algorithms. The second signal in three source signals is standard EEG signal and the theoretical optimal time delay is 193 . The signal is known by us, so the comparison result is persuasive.

Our algorithm is compared with other three algorithms. The parameters of Shi's algorithm are set as follows. The initial value of $b$ is 1 . $\mu_{w}$ is equal to 0.5 and $\mu_{b}$ is equal to 0.001. The comparison result is shown in Fig. 3 .

The parameter $\mu_{b}$ is changed to 0.3 . Other parameters remain unchanged. Another comparison result is shown in

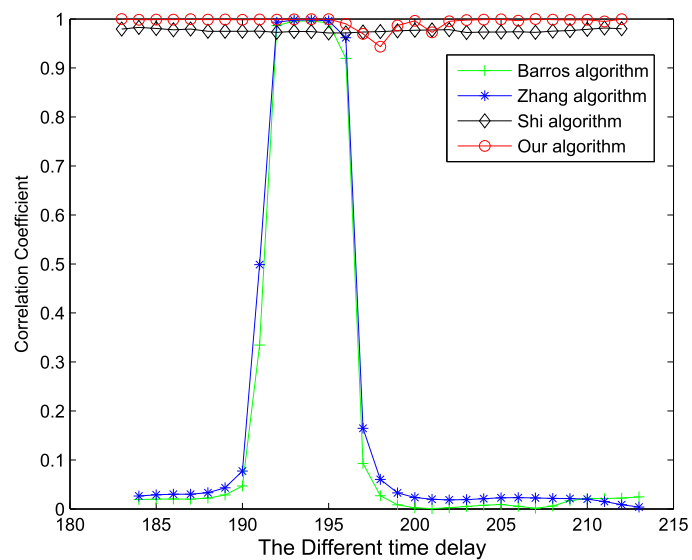

Fig. 3 The correlation coefficients of the estimated ECG signal at the different time delay $\tau$ and the standard ECG signal in different algorithms when $\mu_{b}$ is equal to 0.001

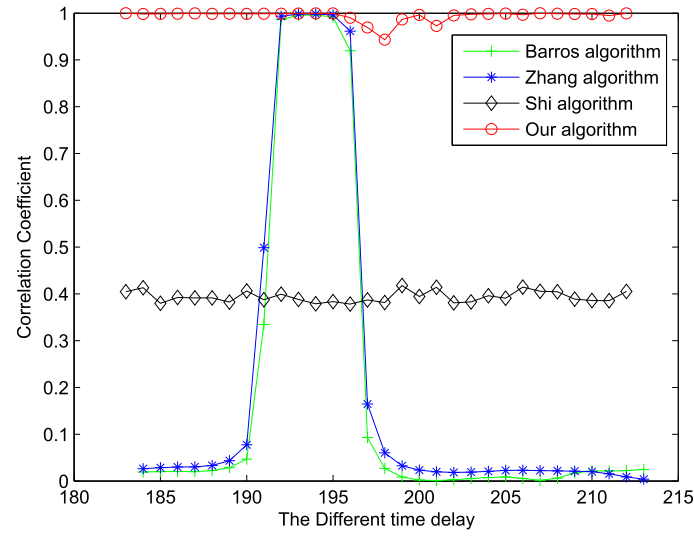

Fig. 4 The correlation coefficients of the estimated ECG signal at the different time delay and the standard ECG signal in different algorithms when $\mu_{b}$ is equal to 0.3

Fig. 4.

According to Fig. 3 and Fig. 4, the following conclusions can be obtained. We can find that our algorithm has better robustness than Barros's algorithm and Zhang's algorithm when the time delay can't be estimated well. Shi's algorithm has good robustness when the parameters are chosen well. But the performance of Shi's algorithm is poor without proper parameters. Therefore, our algorithm has better stability and owns superior extraction accuracy.

In order to demonstrate the practicability of our algorithm, the simulations of real-world ECG data which are shown in Fig. 5 need to be made. The well-known ECG data are measured from a pregnant woman and shared by De Moor. The ECG data are mixed with maternal electrocardiogram (MECG), fetal electrocardiogram (FECG), breathing artifact, electrode artifact and some other noise.

When the FECG signal is the desired signal, the theoretical optimal time delay is 112. Four FECGs are extracted at the optimal time delay by Barros's algorithm, Zhang's algorithm, Shi's algorithm and our algorithm. The extracted results are shown in Fig. 6. The parameters of Shi's algo- 


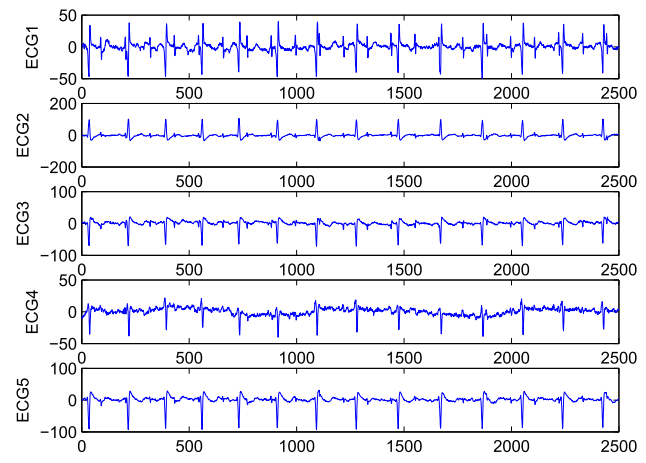

Fig.5 Real ECG signal data measured from a pregnant woman and shared by De Moor

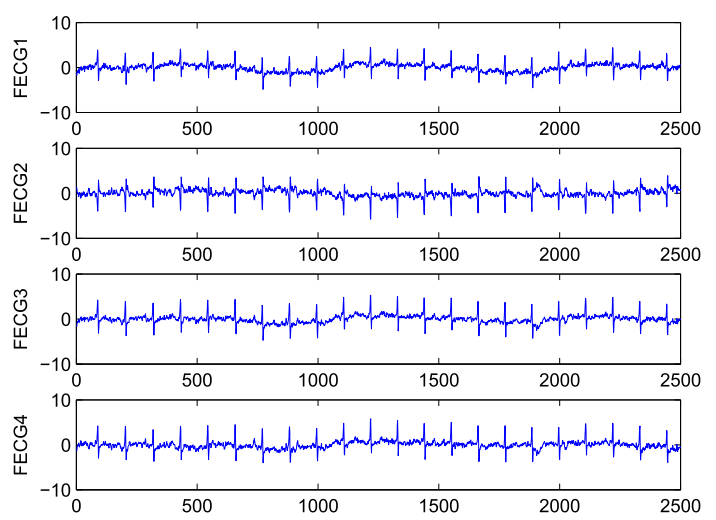

Fig. 6 Comparison of the extracted FECGs at the time delay $\tau=112$. FECG1 is extracted by Barros's algorithm. FECG2 is extracted by Zhang's algorithm. FECG3 is extracted by Shi's algorithm. FECG4 is extracted by our algorithm
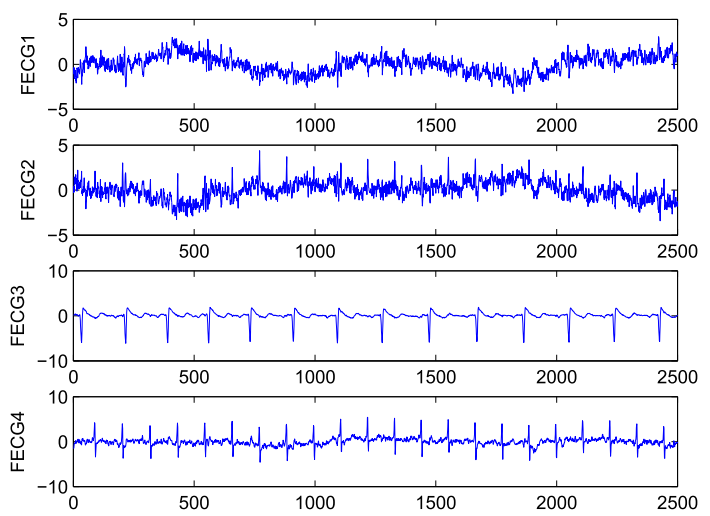

Fig. 7 Comparison of the extracted FECGs at the time delay $\tau=118$. FECG1 is extracted by Barros's algorithm. FECG2 is extracted by Zhang's algorithm. FECG3 is extracted by Shi's algorithm. FECG4 is extracted by our algorithm

rithm are chosen based on [8].

However, the estimated time delay may own error. In order to obtain more visible comparison, four FECGs are extracted at the time delay $\tau=118$ by Barros's algorithm, Zhang's algorithm, Shi's algorithm and our algorithm. The extracted results are shown in Fig. 7.
According to Fig. 6 and Fig. 7, we can know our algorithm is still applicable to real-world data and owns robustness and superior extraction performance.

\section{Conclusion}

We have proposed a BSE algorithm for the extraction of temporal structure signals, such as FECG signals. First, we introduced an objective function based on the probability distribution of the innovation of the desired signal and the time-correlation of the signal with temporal structure. The desired signal is extracted by a fixed-point algorithm derived from the objective function. Furthermore, simulation experiments showed that the proposed algorithm is very robust to the estimation error of the time delay and its performance isn't influenced by parameters, which makes it more suitable to practical application. Finally, we conclude that the algorithm of this paper can be used to solve other signal processing problems.

\section{Acknowledgments}

This work is supported by the Nation Nature Science Foundation of China (No.61301095, No.51509049), the Heilongjiang Province Natural Science Foundation (No.F201345) and the Fundamental Research Funds for the Central Universities of China (No.HEUCF150812/150810).

\section{References}

[1] L.B. Almeida, "Separating a real-life nonlinear image mixture," J. Machine Leaming Research, vol.6, pp.1199-1229, 2005.

[2] K.T. Herring, A.V. Mueller, and D.H. Staelin, "Blind separation of noisy multivariate second-order statistics: remote sensing applications," IEEE Trans. Geosci. Remote Sens., vol.47, no.10, pp.3406-3415, 2009.

[3] J.-Y. Huang, H.-X. Pan, S.-H. Bi, and X.-W. Yang, "Application of particle swarm optimization blind source separation technology in fault diagnosis of gearbox," Journal of Central South Univ. of Tech., vol.15, no.2, pp.409-415, 2008.

[4] M. Cobos and J.J. Lopez, "Two-microphone separation of speech mixtures based on interclass variance maximizationm," Journal of the Acoustical Society of America, vol.127, no.3, pp.1661-1672, 2010.

[5] J. Gao, X. Zhu, and A.K. Nandi, "Independent component analysis for multiple-input multiple-output wireless communication systems," Signal Processing, vol.91, no.4, pp.607-623, 2011.

[6] A.K. Barros and A. Cichocki, "Extraction of specific signals with temporal structure," Neural Computation, vol.13, no.9, pp.1995-2003, 2001.

[7] Z.-L. Zhang and Z. Yi, "Robust extraction of specific signals with temporal structure," Neural Computation, vol.69, no.7-9, pp.888-893, 2006.

[8] Z.W. Shi and C.S. Zhang, "Semi-blind source extraction for fetal electrocardiogram extraction by combining non-Gaussianity and time-correlation," Neurocompution, vol.70, no.7-9, pp.1574-1581, 2007.

[9] C.L. Li and G.S. Liao, "Notes on two temporal structure based methods for blind extraction of fetal electrocardiogram," Neural Computing and applications, vol.21, no.7, pp.1647-1650, 2012.

[10] A.W. Van der Vaart, Asymptotic atatistics, London, Cambrige University Press, 1998. 
[11] A. Hyvarinen, J. Karhunen, and E. Oja, Independent Component Analysis, New York, Wiley, 2001.

[12] A. Hyvärinen, "Complexity pursuit: separating interesting components from time-series," Neural Computation, vol.13, no.4, pp.883-898, 2001.

[13] W. Girolami, "An alternative perspective on adaptive independent component analysis algorithm," Neural Computation, vol.10, no.8, pp.2103-2114, 1998.

[14] T.-W. Lee, M. Girolami, and T.J. Sejnowski, "Independent component analysis using an extended informax algorithm for mixed aubgaussion and supergaussion sources," Neural Computation, vol.11, no.2, pp.417-441, 1999.

[15] A. Hyvarinen, "Fast and robust fixed-point algorithm for independent analysis," IEEE Trans. Neural Netw., vol.11, no.7, pp.1739$1768,1999$.

[16] http://www.bsp.brain.riken.jp/ICALAB/ICALABSignalProc/

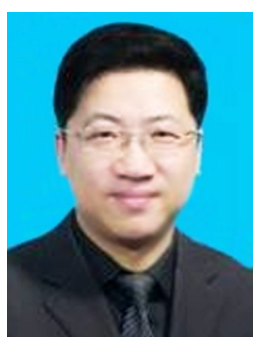

Yibing Li received the B.S. and M.S. and Ph.D. degrees in Harbin Marine engineering college, Harbin engineering university in 1989, 1997 and 2003, respectively. He has been a teacher in Harbin Engineering University of China since 1989, and became as professor in 2004. During 2007-2008, he stayed in the University of Hong Kong Electronic Engineering lab as a visiting scholar. Now he is a IEEE member, a senior member of China Institute of Computer Federation Communications and a senior member of China

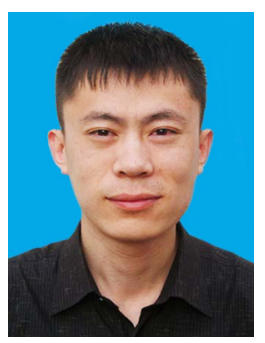

Wei Nie received the B.S. in Communication Engineering from Harbin Engineering University (HEU) in 2013. During 2013-2014, he stayed in HEU to read M.S., During 2014-2015, he stayed in HEU to read P.D.. He is a student member of Institute of Electronics, Information and Communication Engineers (IEICE). Now he is studying communication signal processing.

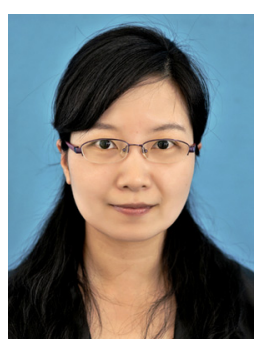

Fang Ye received the B.S. and Ph.D. degrees in Electrical Information Engineering from Harbin Engineering University (HEU) in 2002 and 2006, respectively. She has been a teacher in Harbin Engineering University of China since 2002, and became as associate professor in 2007. During 2007-2008, she stayed in School of Electronics and Computer Science from University of Southampton as a visiting scholar. Now she is a IEEE member, a memmember of China Computer Federation. 